\title{
Simultaneous All-optical 1's Complement Cum Division-by-two Schemes
}

\section{Dilip Kumar Gayen ( $\nabla$ dilipgayen@yahoo.com )}

College of Engineering \& Management, Kolaghat

\section{Research Article}

Keywords: Optical signal processing, Optical arithmetic operation, Optical logic

Posted Date: July 13th, 2021

DOI: https://doi.org/10.21203/rs.3.rs-648222/v1

License: (c) (i) This work is licensed under a Creative Commons Attribution 4.0 International License. Read Full License 


\title{
Simultaneous all-optical 1's complement cum division-by-two schemes
}

\author{
Tanay Chattopadhyay ${ }^{1}$ and Dilip Kumar Gayen ${ }^{2, *}$ \\ ${ }^{1}$ Mechanical Operation (Stage-II), Sagardighi Thermal Power Project, WBPDCL, \\ Manigram, Sagardighi, 742237, West Bengal, India. (email: tanay2222@ rediffmail.com). \\ ${ }^{2}$ Department of Computer Science and Engineering, College of Engineering and \\ Management, Kolaghat, KTPP Township, Purba Medinipur, 721171, W.B, India \\ (*Corresponding author e-mail: dilipgayen@yahoo.com).
}

\begin{abstract}
Odd and even number detection is an important mathematical operation. Generally when any number divisible by 2 then it is called even number, otherwise it is odd number. Division by 2 can be easily obtained by putting a point before least significant bit (LSB) of any binary number. As an example a number $(27)_{10}=(11011)_{2}$ when divided by 2 its result will be $(1101.1)_{2}=(13.5)_{10}$. Hence when we find the fractional bit as logic-1 we can say that the number is odd, otherwise it is even. This operation can be obtained by using a demultiplexer. Here we have developed an optical circuit which can divide any binary integer number by 2, apart from that its 1 's complement can also be obtained from the circuit. Both of the result can be obtained simultaneously. Terahertz optical asymmetric demultiplexer (TOAD) based generally switch assumes a vital part to plan this n-bit circuit. Numerical simulations are done to urge the exhibition of the circuit.
\end{abstract}

Keywords: Optical signal processing; Optical arithmetic operation; Optical logic.

\section{Introduction}

Optics shows potential option of electronic calculation as of late. Because of high transfer speed and less information transport necessity, optical information preparing and correspondence become better known [1]. Semiconductor optical intensifier (SOA) makes exchanging activity all the more simple and rapid. Optical sign preparing interferometric switches to construct the upset. Among different switches, TOAD be a solitary arm interferometer. TOAD is capable of deal with information processing about $1 \mathrm{~Tb} / \mathrm{s}$ [2-5]. This 
switch has quick shift time, low power consumption, low latency and noise. Again this switch has very high nonlinear properties with thermally stabile utilized in correspondence thoroughly [2-4]. The multipliers which are utilizing two diverse plan arrangements come within reach have been designed by Sharma [5]. K. Maji et al have designed all-optical recurrence encoded AND, OR, and NOT rationale entryways and their presentation reenacted to affirm their practicality [6]. Huo et al [7] proposed a reconfigurable fotonic filter based on TOAD based switch. Maji et al [8] also proposed a dual control TOAD based 2's complement method in their paper. In their other paper they also proposed XOR gate and binary to gray bit conversion using this interferometric switch [9]. Here we have proposed a plan of n-bit binary division- by-2 circuit using TOAD. Generally when any binary number is divided by 2 one fractional bit appears. If the number is odd, fractional bit is logic-' 1 ' otherwise it is logic- ' 0 '. By dividing different numbers by 2 we see that results can be easily obtained by putting a point before the least significant integer bit of any binary number. As an example if we divide a binary number $(11011101)_{2}$ by 2 , the result will be $(1101110.1)_{2}$. To play out this activity we have used TOAD primarily based interferometric switch. This all-optical circuit can perform division-by-2 and also its 1's complement simultaneously.

\section{Theoretical operation of the switch:}

TOAD could be a solitary arm interferometer. It utilizes a SOA that is put unevenly situated in fibre circle. The optical coupler (50:50) is used to join the two end of fibre to form a loop. Here we denote the incoming signal as IS from a CW signal of wavelength $\lambda_{1}$ (generally it is of $1500 \mathrm{~nm}$ ), which divides into two equivalent parts. One is counter clockwise parts and other is clockwise parts. These two sections spread around the circle inverse way to one another and reconsolidate at coupler. Depending upon the segment distinction among clockwise and counter clockwise beats, valuable or damaging obstruction happens. Stage distinction preserves by pertaining beat light emission of wavelength $\lambda_{2}$ from another CW signal (generally $1550 \mathrm{~nm}$ of wavelength). This signal passes through a Ti:LiNbO3 electrooptic modulator driven by NRZ pulse generator. After that it is amplified by erbium doped fiber amplifier (EDFA) followed by variable optical attenuator (VOA). At every stage polarization of the intense pulse can be controlled by polarization controller (PC). The final signal (CP) mixing can be done by a coupler with the other IP clock wise pulse. Coupler is attached in the fibre circle as demonstrated in Fig.1(a). Data signals and Gaussian signal of full-width half maximum $(\mathrm{FWHM})=\sigma$ are applied at almost alike point in time. Semiconductor optical amplifier is put unevenly in the fibre circle. The asymmetric distance 
is $\Delta x=T_{e x} / 2\left(T_{e x}\right.$ is the eccentricity time). Due to this asymmetric counter clockwise and clockwise parts proliferate dissimilar times throughout semiconductor optical amplifier. This timing difference creates different phase between the counter clockwise and clockwise parts. As a result constructive or destructive interference occurs at the input coupler. According light can reach at upper or lower port $[3,4,10]$.

$$
\begin{aligned}
& P_{\text {Upper }}(t)=\frac{P_{I S}}{2}\left\{C(t)+C^{\prime}(t)-2 \sqrt{C(t) \cdot C^{\prime}(t)} \cdot \cos (\Delta \theta)\right\} \\
& P_{\text {Lower }}(t)=\frac{P_{I S}}{2}\left\{C(t)+C^{\prime}(t)+2 \sqrt{C(t) \cdot C^{\prime}(t)} \cdot \cos (\Delta \varphi)\right\}
\end{aligned}
$$

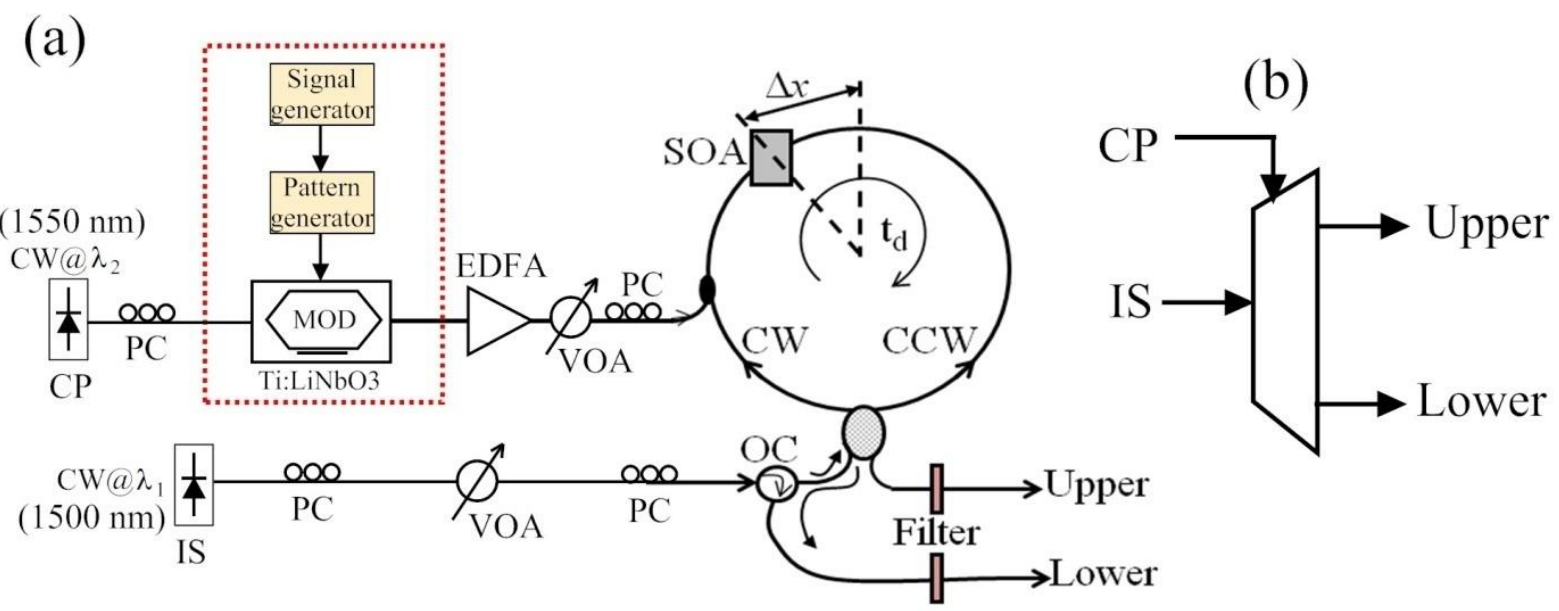

Fig. 1. (a) Optical switch based on TOAD (b) Schematic diagram of TOAD

where, $C(t)$ and $C^{\prime}(t)$ measure the SOA gain for clockwise and anticlockwise signal respectively. Between the counter clockwise and clockwise parts creates a phase difference, which is numerically communicated as [11-15],

$$
\Delta \theta=-\frac{\alpha}{2} \ln \left\{\frac{C(t)}{C^{\prime}(t)}\right\}
$$

Here linewidth enhanced factor is $\alpha$. Here we consider that SOA is wavelength independent and its unsaturated gain $G_{U}$ is [12]

$$
G_{U}=e^{\left[\left[\Gamma g_{d} N_{c}\left(\frac{I \tau_{r}}{e \omega l L N_{c}}-1\right)-r\right]\right.}
$$


Where $g_{d}=$ differential gain, $\Gamma=$ confinement factor, $L=$ active length of SOA, $N_{c}=$ carrier density at transparency, $I=$ biasing current of SOA, $\tau_{r}=$ recovery time of SOA, $e=$ charge of electron, $\omega=$ width of active region of SOA, $l=$ depth of active region of SOA, $\Upsilon$ $=$ SOA internal loss per unit length. When control pulse (CP) is OFF i.e., absence of control pulse, information signal (approaching signal parts $\mathrm{CW}$ and $\mathrm{CCW}$ ) reaches to SOA by dissimilar times. Therefore one of the pulse experience an unsaturated little amplifier acquire $G_{U}$. As a result when the pulses recombine at the input coupler i.e. $C(t)$ is nearly equal to $C^{\prime}(t)$. At that point, $\Delta \theta$ is almost equal to 0 and articulation for $P_{U p p e r}$ is nearly equal to 0 and $P_{\text {Lower }}$ is equal to $P_{I S}(t)$. $G_{U}$. So the input signal is returned back around the source. This returned back pulse can be segregated by a circulator. This circulator is named as optical circulator (OC). When a control pulse is infused into fibre loop, after the time $t_{s}$ it penetrates SOA and alters its benefit as $[11,12]$,

$$
G(t)=\frac{1}{1-\left(1-\frac{1}{G_{U}}\right) \cdot e^{\left(-\frac{E_{c p}(t)}{E_{s a t}}\right)}}
$$

$E_{c p}(t)$ is control pulse energy.

When $\mathrm{CP}$ is infused into the fibre loop then the gain of SOA decreases rapidly as $G^{\prime}(t)=e^{f(t)}$ and $f(t)$ can be determined by the ordinary differential equation as [12],

$$
\frac{d f(t)}{d t}=\frac{\Gamma g_{d} N_{c} L\left(\frac{I \tau_{r}}{e \omega l L N_{c}}-1\right)-f(t)}{\tau_{r}}-\frac{P_{i n}(t)}{E_{s a t}}\left(e^{f(t)}-1\right)
$$

Then, $\Delta \theta$ is nearly equal to $-\pi$ then the information egress from the upper line i.e. $P_{\text {Upper }}(t)$ is equal to 0 and $P_{\text {Lower }}(t)$ is not 0 , the comparing esteems can be gotten from the conditions (1) and (2), separately. In a short time the gain of SOA recuperates owing to carrier insertion into SOA by recovery time $\tau_{r}$. The energy of the incoming signal is about one-tenth time less than that of the control pulse, A band pass filter alter ought to be utilized at the yield of TOAD based change to dismiss the $\mathrm{CP}$ and pass the IS. The block diagram of a TOAD is appeared in Fig.1(b). For optimum performance of TOAD based interferometic switch must follow the relation: $\sigma<T_{e x}<0.5 T_{c}<\tau_{r}<1.5 T_{c}$, where $T_{c}$ is the cycle period.

\section{Division-by-2 cum 1's complement}


In the above section we notice when $\mathrm{CP}$ is applied to TOAD, at that point IS is communicated to upper line. At that time, no information is found at the lower line. If control pulse is absent, the IS is coordinated to the lower line and at that time no information is exposed to the upper line. We can plan $n$-bit division-by-2 circuit utilizing $\mathrm{n}$ no. of TOADs that is appeared in the Fig.2. In this communication we utilize all the output line (upper and lower) of the TOADs. $\left(A_{n} A_{n-1} \ldots A_{1} A_{0}\right)_{2}$ are n-bit inputs of wavelength $\lambda_{1}$. Upper ports yield all TOADs structures division-by-2 yield as $\left(\mathrm{D}_{\mathrm{n}} \mathrm{D}_{\mathrm{n}-1} \ldots \mathrm{D}_{1} \mathrm{D}_{0} \mathrm{D}_{-1}\right)_{2}$, where $\mathrm{D}_{-1}$ is the fractional part. Likewise $\left(\mathrm{C}_{\mathrm{n}} \mathrm{C}_{\mathrm{n}-\ldots} \ldots \mathrm{C}_{1} \mathrm{C}_{0}\right)_{2}$ are the 1's complement, which are taken from lower lines of all TOADs. IP of wavelength $\lambda_{2}$ are supplied from steady light source.

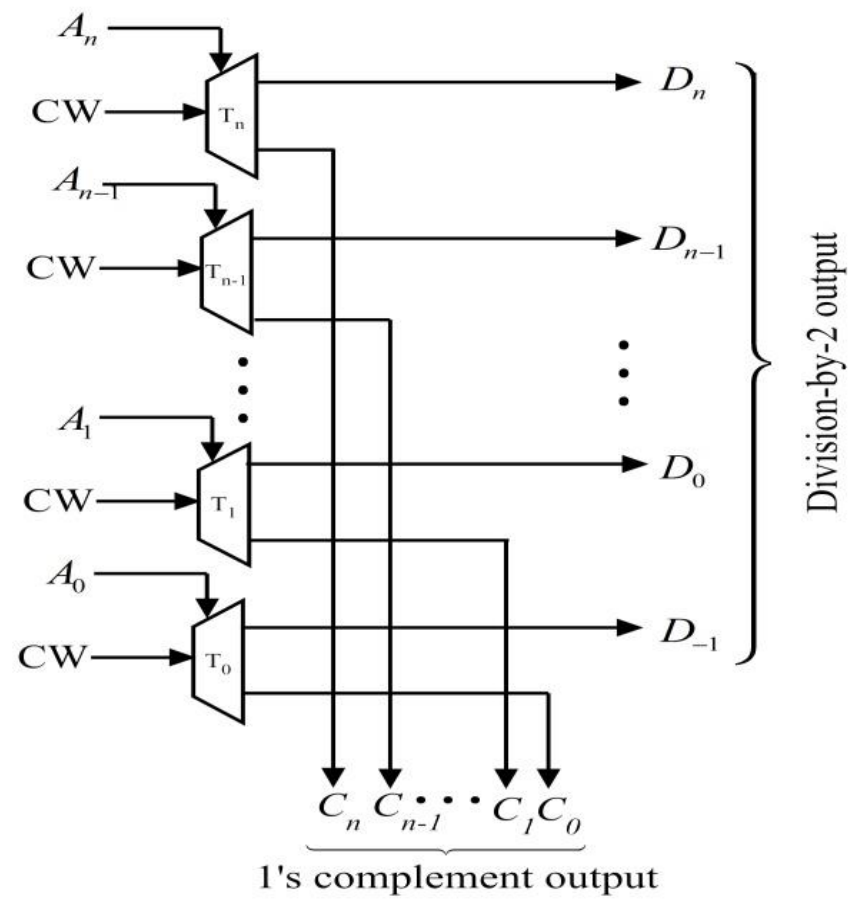

Fig. 2. All-optical division-by-2 cum 1's complement conversion circuit using TOAD. $\left(A_{n} A_{n-}\right.$ $\left.{ }_{1} \ldots A_{1} A_{0}\right)_{2}$ is input number, division-by-2 output is $\left(\mathrm{D}_{n} \mathrm{D}_{\mathrm{n}-1} \ldots \mathrm{D}_{0} \mathrm{D}_{-1}\right)_{2}$, where $\mathrm{D}_{-1}$ is fractional bit, $\left(\mathrm{C}_{\mathrm{n}} \mathrm{C}_{\mathrm{n}-1} \ldots \mathrm{C}_{1} \mathrm{C}_{0}\right)_{2}$ is 1 's complement output.

The activity of the proposed circuit can be seen with any problem. One test set makes the activity clear to each one. Let us consider we have a 4-digit information $\left(\mathrm{A}_{3} \mathrm{~A}_{2} \mathrm{~A}_{1} \mathrm{~A}_{0}\right)_{2}=$ (1011)2. Now our task is to convert is information to its equivalent to 1's complement and furthermore divide it by 2 . These info pieces are given as CP to TOADs $\left(\mathrm{T}_{0}, \mathrm{~T}_{1}, \mathrm{~T}_{2}\right.$ and $\left.\mathrm{T}_{3}\right)$. At that point as indicated by the exchanging rule of TOAD, CW information of TOADs $\mathrm{T}_{0}$, 
$\mathrm{T}_{1}$ and $\mathrm{T}_{3}$ are sent to the upper line. Additionally in TOAD $\left(\mathrm{T}_{2}\right)$, information corresponds to the lower line. Henceforth we acquire yields $\left(\mathrm{D}_{2} \mathrm{D}_{1} \mathrm{D}_{0} \mathrm{D}_{-1}\right)_{2}=(101.1)_{2}$ and $\left(\mathrm{C}_{3} \mathrm{C}_{2} \mathrm{C}_{1} \mathrm{C}_{0}\right)_{2}=$ $(0100)_{2}$, which are the division-by-2 what's more, 1's complement of the information parallel information (1011)2 separately.

\section{Results and discussion}

Numerical simulation with MATLAB has been carried out using different parameters used in simulations and experiment of various papers [15-17]. The estimations of the different boundaries utilized in this reproduction are as per the following: $\mathrm{I}=400 \mathrm{~mA}, \Gamma=0.48, g_{d}=$ $3.3 \times 10^{-20} \mathrm{~m}^{2}, \quad N_{c}=1.0 \times 10^{24} \mathrm{~m}^{-3}, \omega=1.5 \mu \mathrm{m}, l=250 \mathrm{~nm}, L=1500 \mu \mathrm{m}, \Upsilon=2700 \mathrm{~m}^{-1}$, unsaturated amplifier gain of the $\mathrm{SOA}\left(G_{U}\right)=30 \mathrm{~dB}$, acquire recuperation season of the SOA $\left(\tau_{r}\right)=50 \mathrm{ps}$, immersion energy of the SOA $\left(E_{s a t}\right)=1000 \mathrm{fJ}$, unconventionality of the circle $\left(T_{e x}\right)=15 \mathrm{ps}$, FWHM of the control pulse $(\sigma)=3.6 \mathrm{ps}$, line-width upgrade factor $(\alpha)=6$, cycle period $\left(T_{C}\right)=50 \mathrm{ps}$, and a control pulse energy $\left(E_{c p}\right)=100 \mathrm{fJ}$. The input and output waveforms for six sets of data are given in Fig. 3(a) to 3(f). Parallel input $\left(\mathrm{A}_{7} \mathrm{~A}_{6} \mathrm{~A}_{5} \mathrm{~A}_{4} \mathrm{~A}_{3} \mathrm{~A}_{2} \mathrm{~A}_{1} \mathrm{~A}_{0}\right)_{2}$ is given to the circuit as $\mathrm{CP}$ of the TOADs $\mathrm{T}_{7}-\mathrm{T}_{0}$ individually. Simultaneous outputs are obtained at the $\mathrm{O} / \mathrm{P}$ ports parallel manner. Hence the $\mathrm{O} / \mathrm{P}$ $\left(\mathrm{D}_{6} \mathrm{D}_{5} \mathrm{D}_{4} \mathrm{D}_{3} \mathrm{D}_{2} \mathrm{D}_{1} \mathrm{D}_{0} \mathrm{D}_{-1}\right)_{2} \quad$ check consequences of division-by-2 furthermore, $\left(\mathrm{C}_{7} \mathrm{C}_{6} \mathrm{C}_{5} \mathrm{C}_{4} \mathrm{C}_{3} \mathrm{C}_{2} \mathrm{C}_{1} \mathrm{C}_{0}\right)_{2}$ check the $1 \mathrm{~s}$ complement activity for the given information. We also plot the gain variation with the used input data for 8-TOAD based interferometric switch in Fig.4.

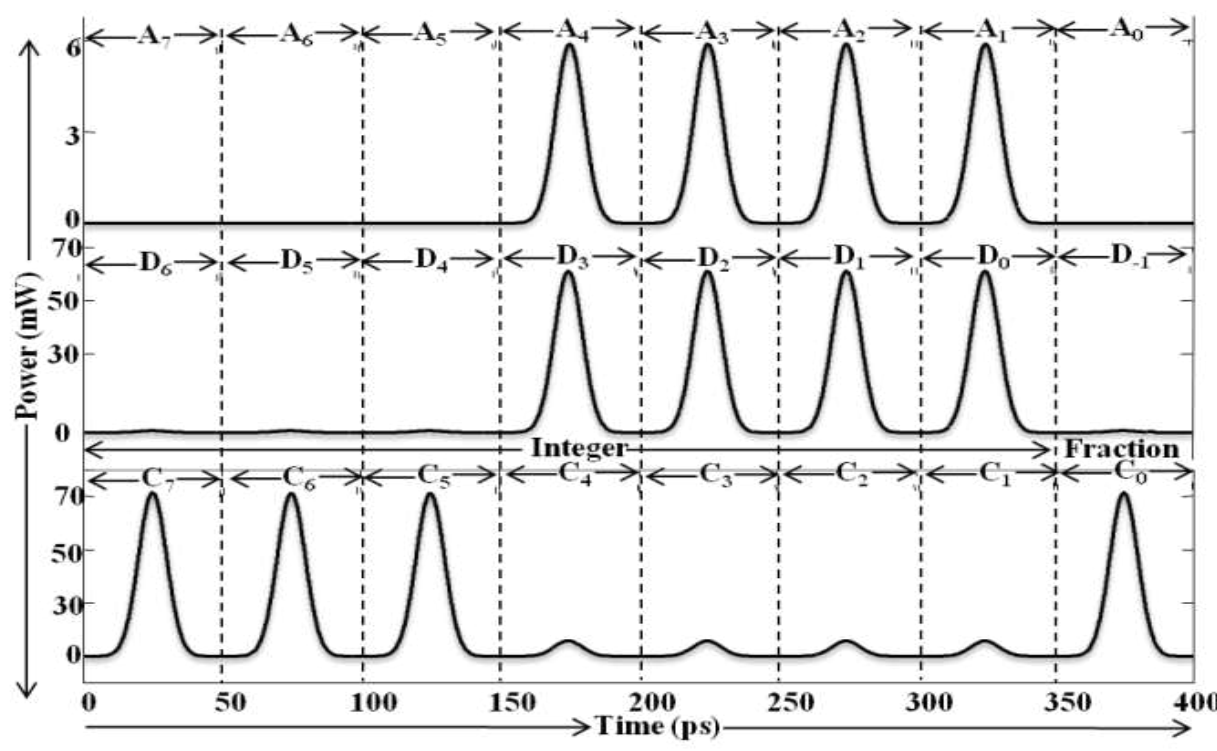




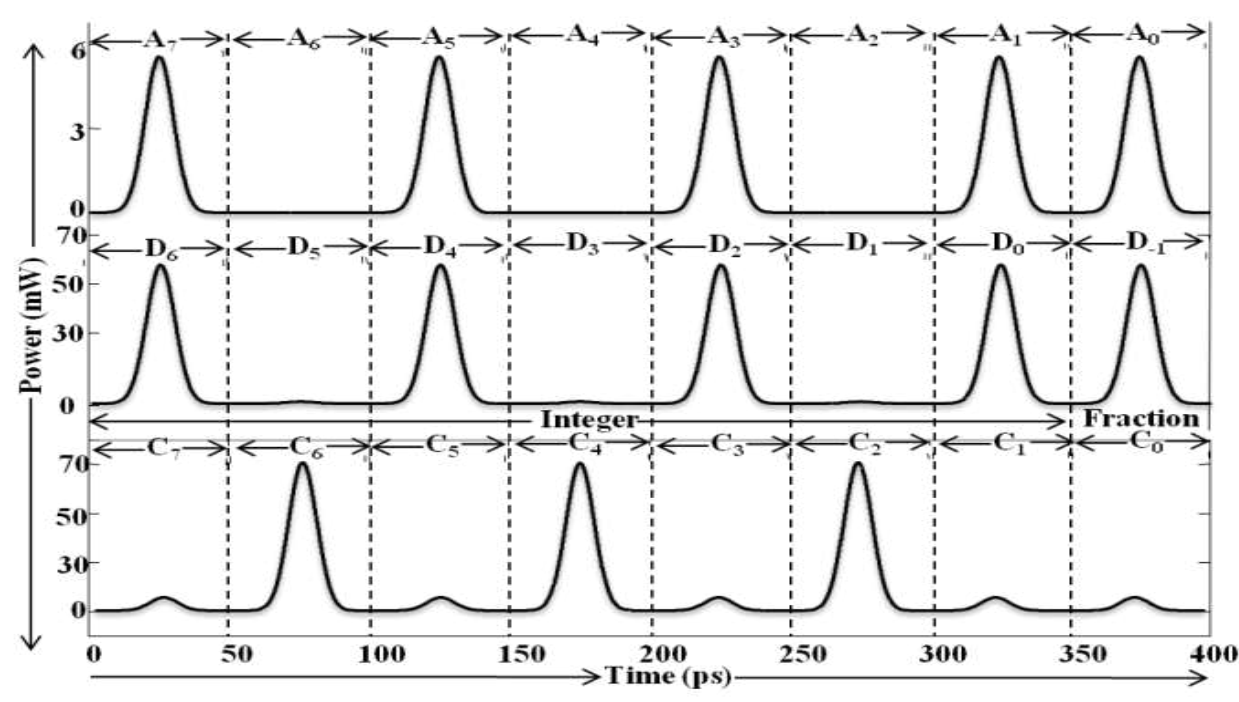

(b)

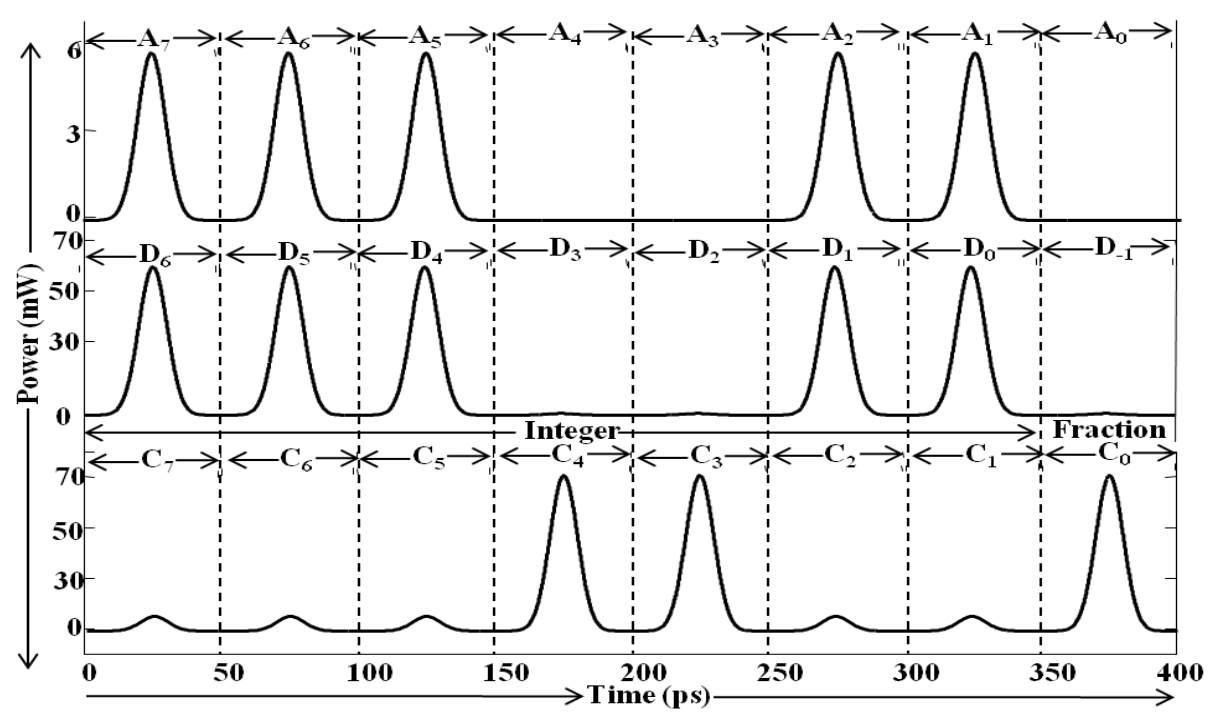

(c)

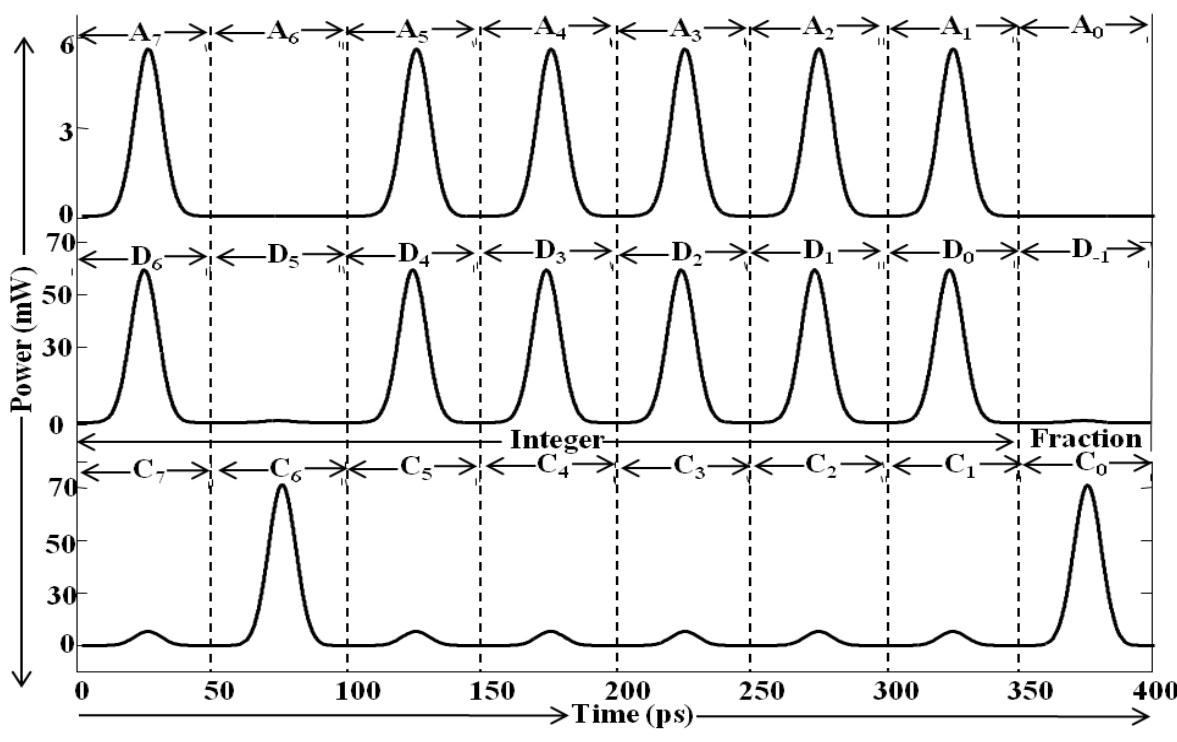

(d) 


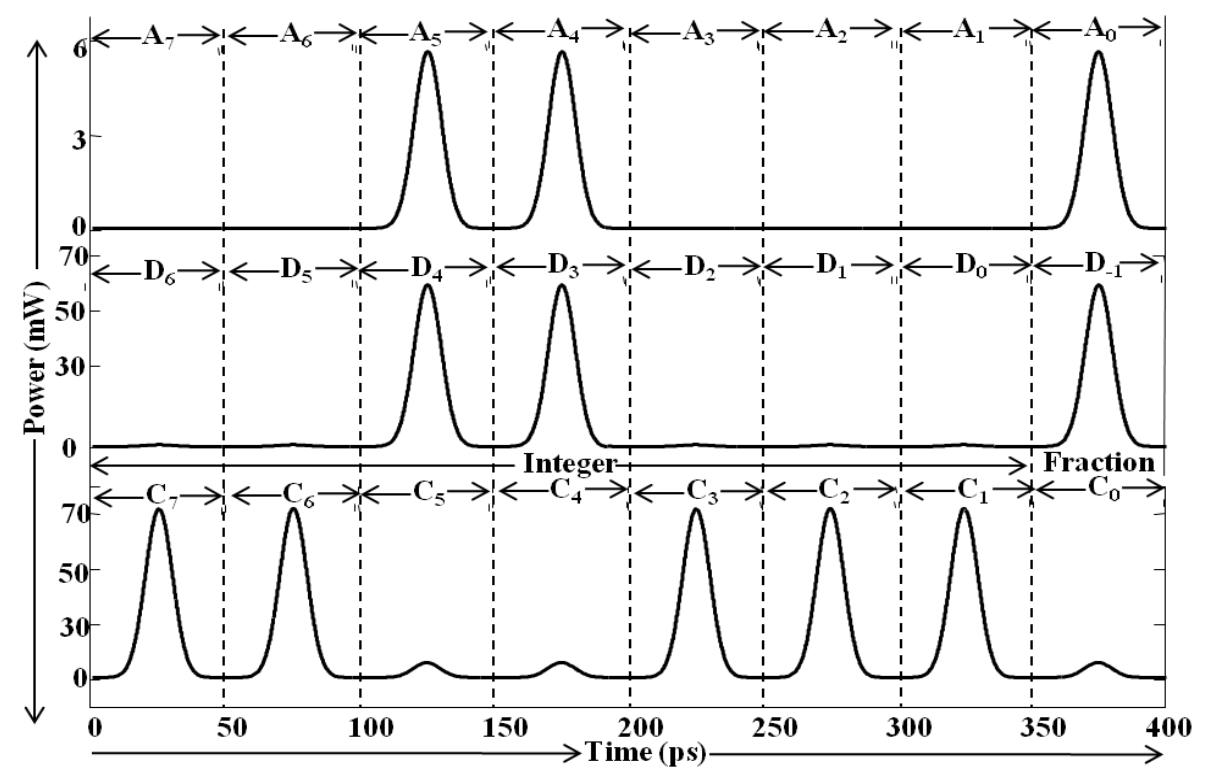

(e)

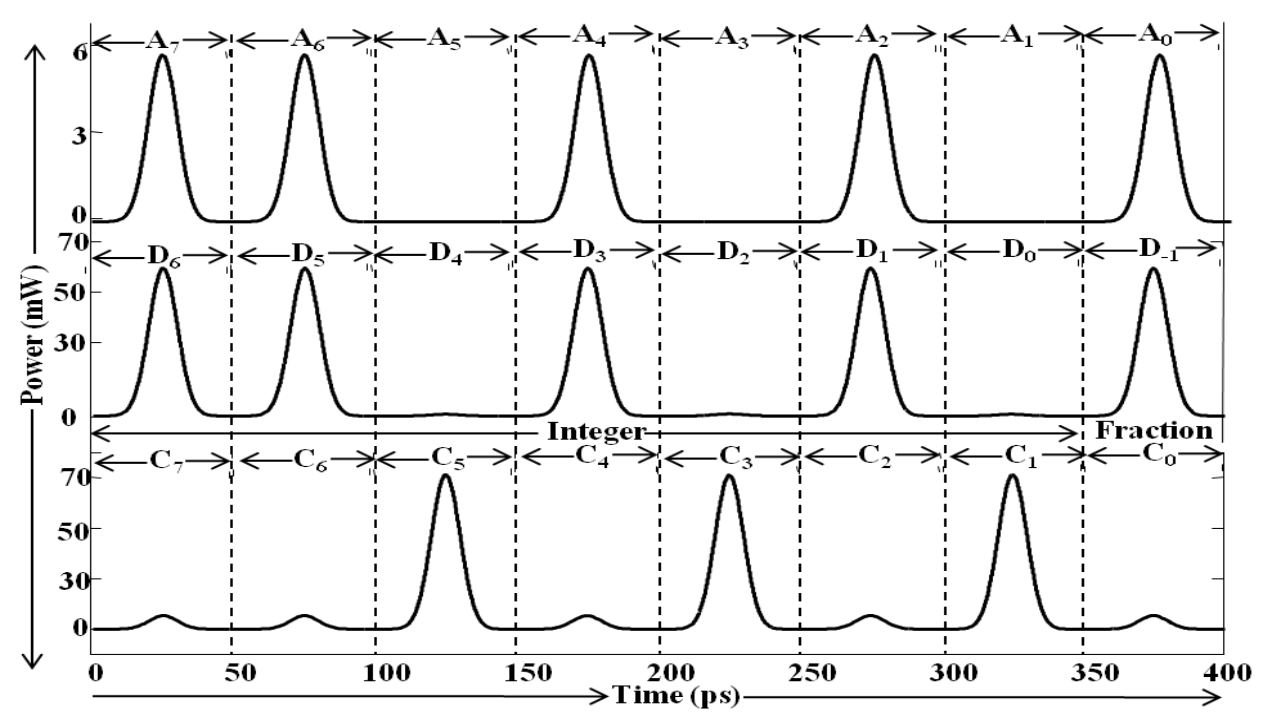

Fig. 3. Simulated input and output waveforms, (a) input is $(00011110)_{2}$, division-by-2 output is $(0001111.0)_{2}$ and 1's complement output is $(11100001)_{2}$, (b) input is $(10101011)_{2}$, divisionby-2 output is (1010101.1)2 and 1's complement output is $(01010100)_{2}$, (c) input is (11100110)2, division-by-2 output is $(1110011.0)_{2}$ and 1's complement output is $(00011001)_{2}$, (d) input is $(10111110)_{2}$, division-by-2 output is (1011111.0) 2 and 1's complement output is $(01000001)_{2}$, (e) input is $(00110001)_{2}$, division-by-2 output is $(0011000.1)_{2}$ and 1 's complement output is $(11001110)_{2}$, (f) input is $(11010101)_{2}$, division-by-2 output is $(1101010.1)_{2}$ and 1's complement output is $(00101010)_{2}$. 
To quantify our designed, we figure the extinction ratio (ER) as [13],

$$
E R(\text { in } \mathrm{dB})=10 \log \left(\frac{P_{\min }^{1}}{P_{\max }^{0}}\right)
$$

where $\mathrm{P}^{1}{ }_{\text {min }}$ and $\mathrm{P}^{0}{ }_{\text {max }}$ is the base and most extreme pinnacle force of the ' 0 states also, ' 1 states, separately. For superior, ER ought to be at any rate in the request for $8.5 \mathrm{~dB}$. ER value tells that ' 1 states can be obviously recognized from the ' 0 ' states. The circuit performs depend on SOA acquire recuperation and energy of CP.

The effect of the addition recuperation and energy of CP on the ER is delineated in Fig.5. It is observed that ER increments with expanding energy of $\mathrm{CP}$ and acquire recuperation time up to a specific worth $(\sim 100 \mathrm{fJ}$ and $\sim 100 \mathrm{ps})$ after which ER is diminished. It is likewise seen from Fig. 5, high addition recuperation time and the energy of $\mathrm{CP}$ decreases the ER. This justified by acquire dynamic reaction of SOA. For high addition recuperation time, beat requires more opportunity to recuperate its underlying increase which decreases the ER. The immersion energy lessens with diminish of gain recuperation time for fixed immersion power. That is the reason less energy is needed to soak the SOA.

A fundamental boundary that influences the exhibition of circuit is that the component of the optical pulse. Fig.6 shows the impact of gain recuperation time and input data measurement on the ER. It clarifies that the ER diminishes with diminishing heartbeat measurement and gain recuperation time. This happens because of a proceeding with lopsidedness; all together that the SOA needs more to recuperate its benefit and gives the compulsory alteration of segment. The ER becomes steady once some specified cost of gain recuperation time and heartbeat measurement. A specific ER will be accomplished with less energy for a more limited pulse measurement. 


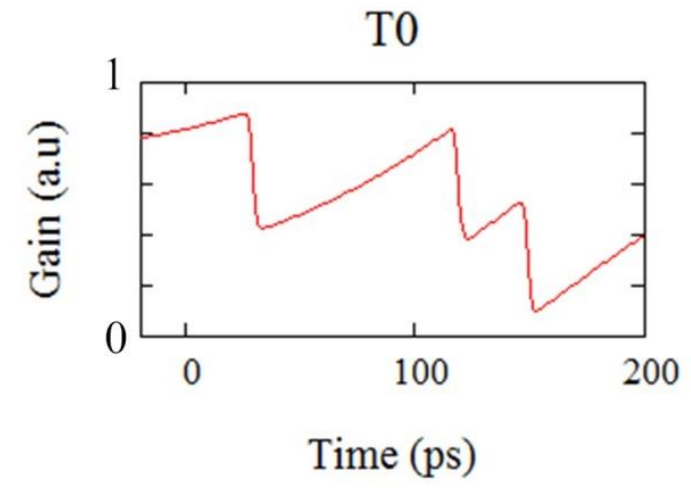

$\mathrm{T} 2$

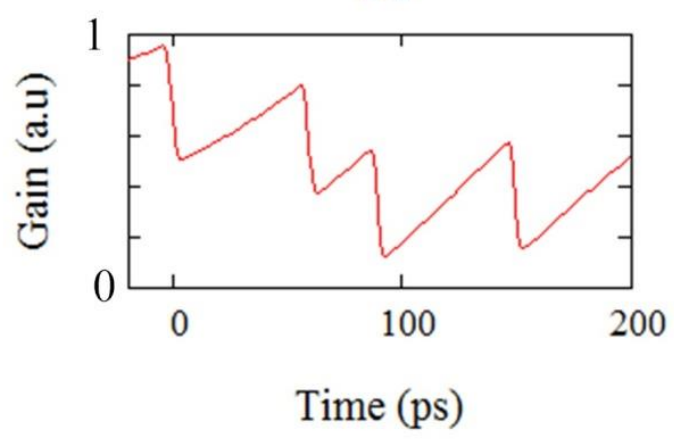

T4

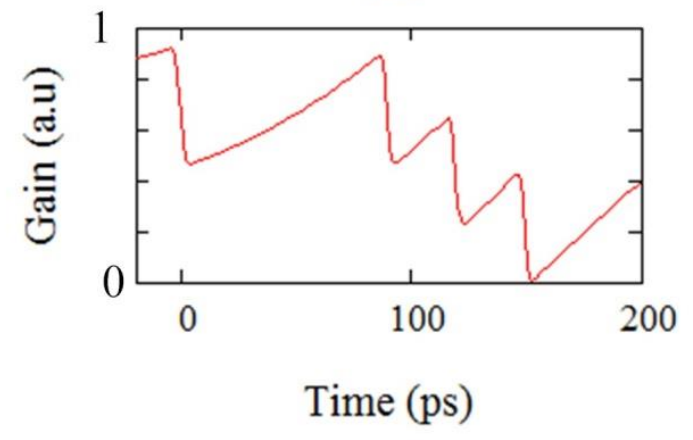

T6

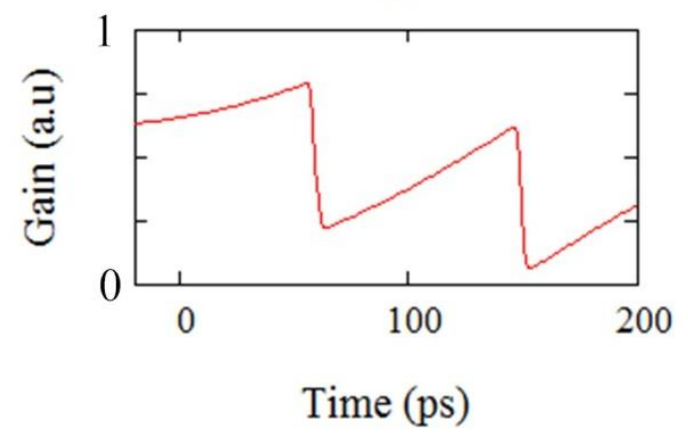

T1

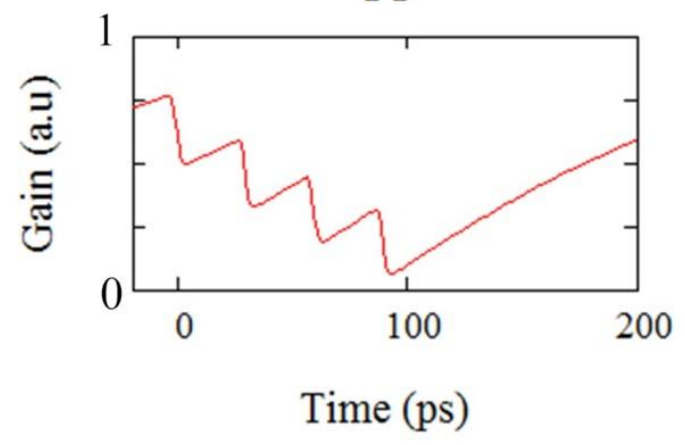

T3

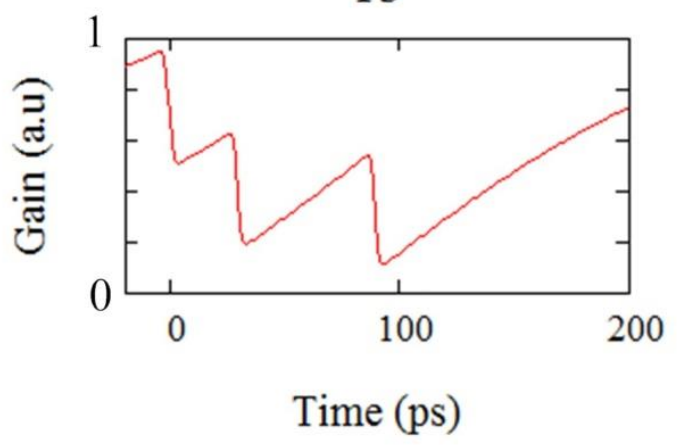

T5

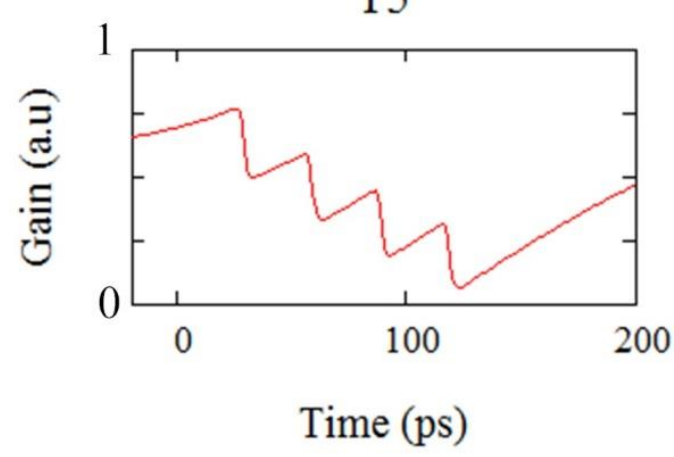

$\mathrm{T} 7$

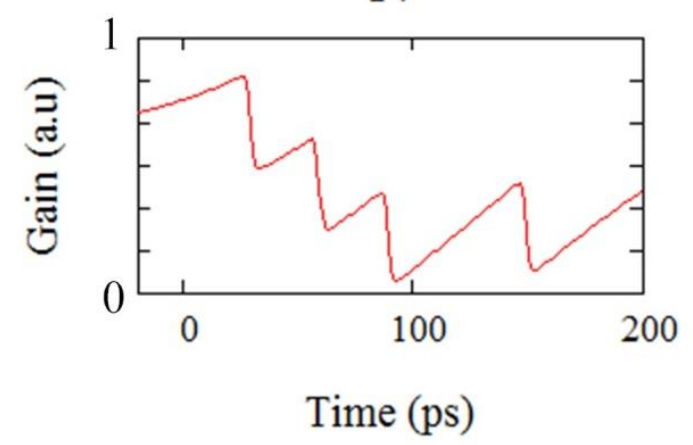

Fig. 4: Simulated gain variation of SOA of TOAD T0 to T7 with used control data pulses. 


\section{ER in dB}

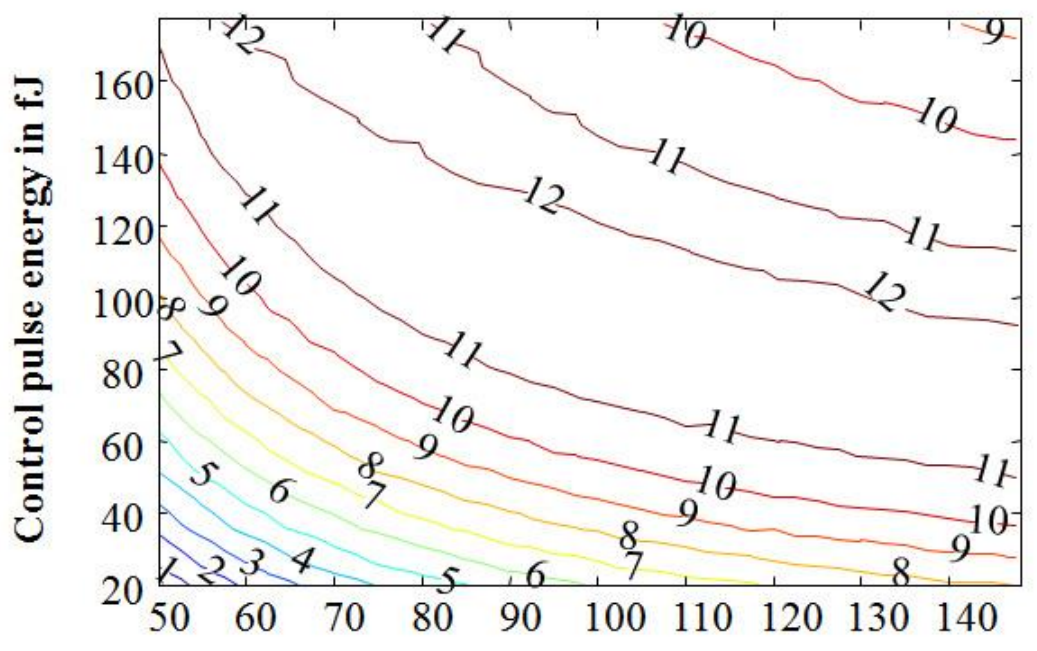

Gain recovery time in ps

Fig. 5. Variation of ER with gain recovery time and energy of $\mathrm{CP}$ at the outputs, while keeping other parameter fixed.

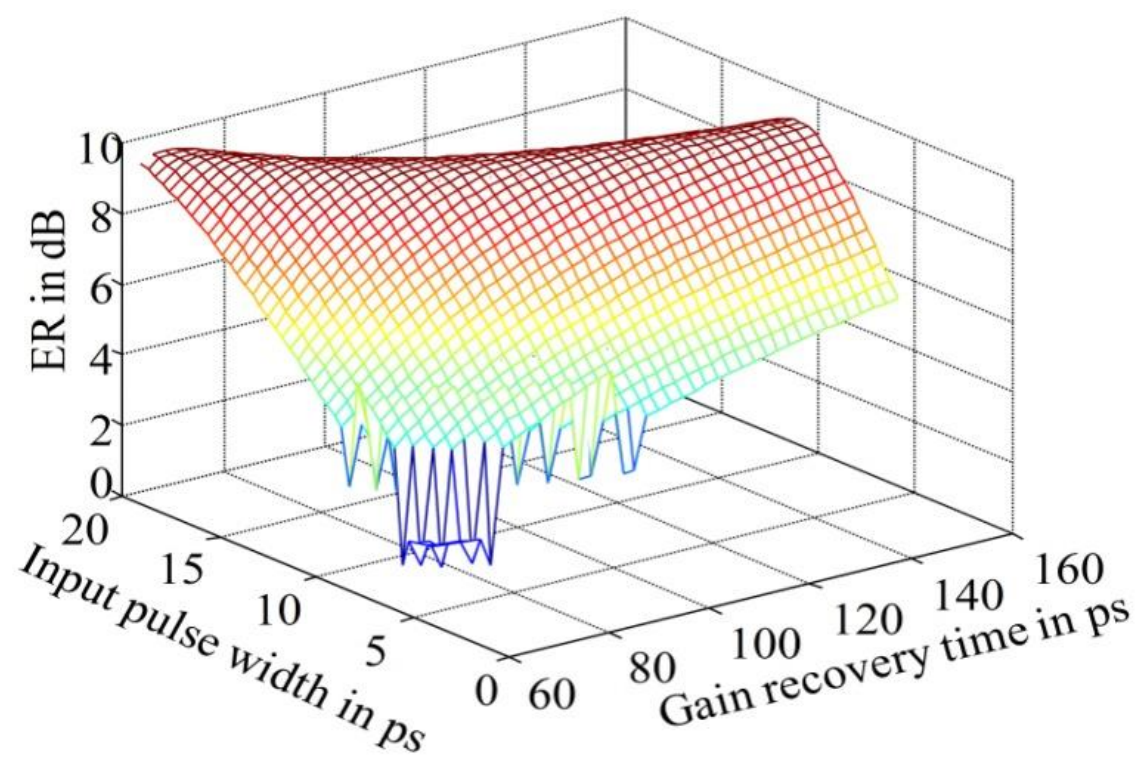

Fig. 6. Variation of ER with pulse width and recovery time at the outputs.

To assess the presentation of the circuit significantly required estimating boundary is contrast ratio (CR). Here, contrast proportion boundary is utilized for the examination. It is the 
effectiveness through which the communicated optical power is balanced over the correspondence connects. It is picked as an another improvement model, communicated as relationship of the power used in sending a rationale level 'one' to the power used in sending rationale 'zero'. This could be portrayed as the proportion of least $\mathrm{O} / \mathrm{P}$ peak power of ' 1 ' to most extreme $\mathrm{O} / \mathrm{P}$ peak power of ' 0 ' in $\mathrm{dB}$ (decibel), defined as

$$
C R(\text { in } \mathrm{dB})=10 \log \left(\frac{P_{\text {mean }}^{1}}{P_{\text {mean }}^{0}}\right)
$$

Where, $\mathrm{P}^{1}$ mean and $\mathrm{P}^{0}{ }_{\text {mean }}$ is that the standard of the stature force of ' 1 ' and ' 0 , respectively. We tend to ascertain the C.R (dB) from condition (7) and it gets $15.62 \mathrm{~dB}$. A significant issue that affects the exhibition of circuit is that the optical pulse width. Fig.7 shows the variety of qualification quantitative connection on pulse broadness and recuperation time. The estimation of $\mathrm{CR}$ is about $15.62 \mathrm{~dB}$ for the specified little sign increase of approximate twenty $\mathrm{dB}$ and the executives pulse energy of about $100 \mathrm{fJ}$, individually. Figure clarifies that the $\mathrm{CR}$ diminishes with diminishing both recuperation time and pulse width. The CR component keeps up consistent once some specified pulse broadness and recuperation time. Henceforth an exact CR esteem gets with less energy for a more limited pulse width. This occurs because of a more limited pulse goes quicker through the SOA upgrading so the fast consumption of transporters and prompting a vessel progress from the lower to the higher immersed acquire values. We tend to thoroughly dole out the value of pulse broadness and gain recuperation time with the goal that the circuit execution is better.

We define amplitude modulation (AM) as

$$
A M(\text { in } \mathrm{dB})=10 \log \left(\frac{P_{\max }^{1}}{P_{\min }^{1}}\right)
$$

where $\mathrm{P}^{1}{ }_{\text {max }}$ and $\mathrm{P}^{1}{ }_{\text {min }}$ is the greatest and least peak power of the 1 -states, separately. Fig. 8 showed the progressions of AM with different energy of $\mathrm{CP}$ and gain recuperation time. The AM is tremendously vulnerable to the varieties of gain recuperation time. Notwithstanding, explicit consideration is taken all together that it ought to be a few specific limit in any case design impacts happen at the output of the circuit. 


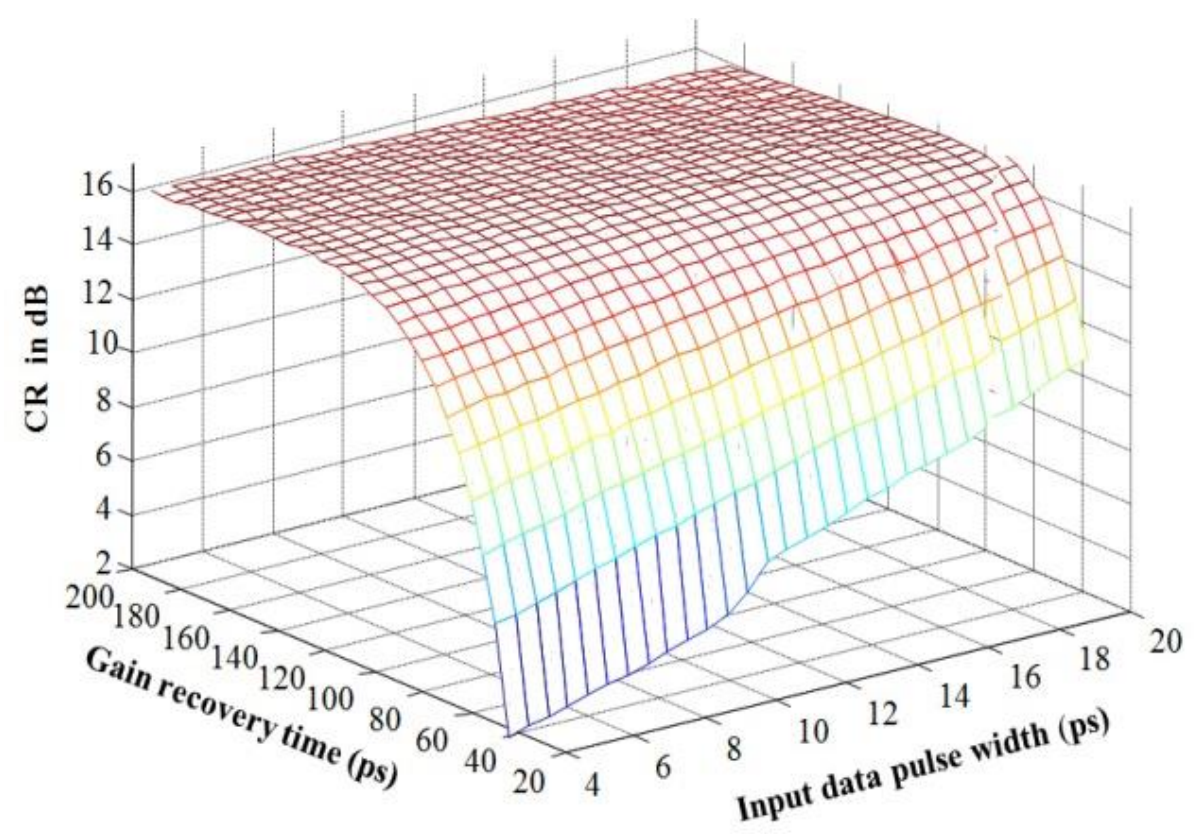

Fig. 7. Variation of CR with pulse width and gain recovery time at the different outputs.

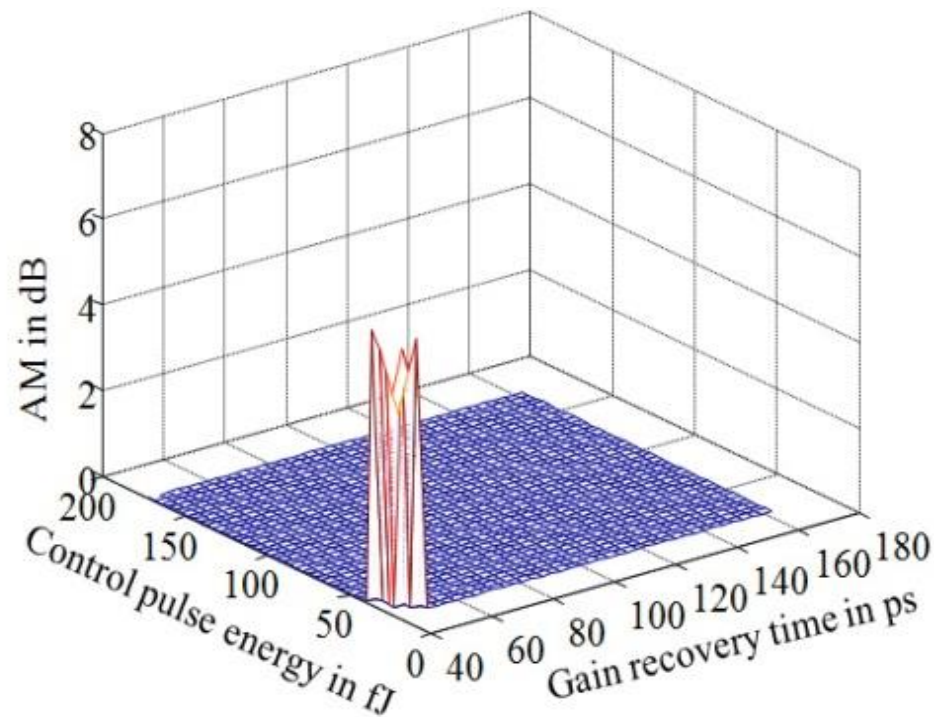

Fig. 8. Variation of AM with gain recovery time and energy of $\mathrm{CP}$ and at the different outputs. 
$Q$ factor is another performing estimating component of the optical circuit. The $Q$ factor can be expressed as,

$$
Q=\frac{P_{\text {mean }}^{1}-P_{\text {mean }}^{0}}{\sigma_{\text {std }}^{1}+\sigma_{\text {std }}^{0}}
$$

where $\sigma_{\text {std }}^{1}$ and $\sigma_{\text {std }}^{0}$ are the standard deviation of the peak power of the 1-states what's more, 0-states, individually. Fig.9 shows the variety of the Q-factor on energy of $\mathrm{CP}$ with different the pulse width. From this figure, we see that Q-factor increments with expanding of the pulse width since it requires considerably more an ideal opportunity to travel SOA by the control pulse.

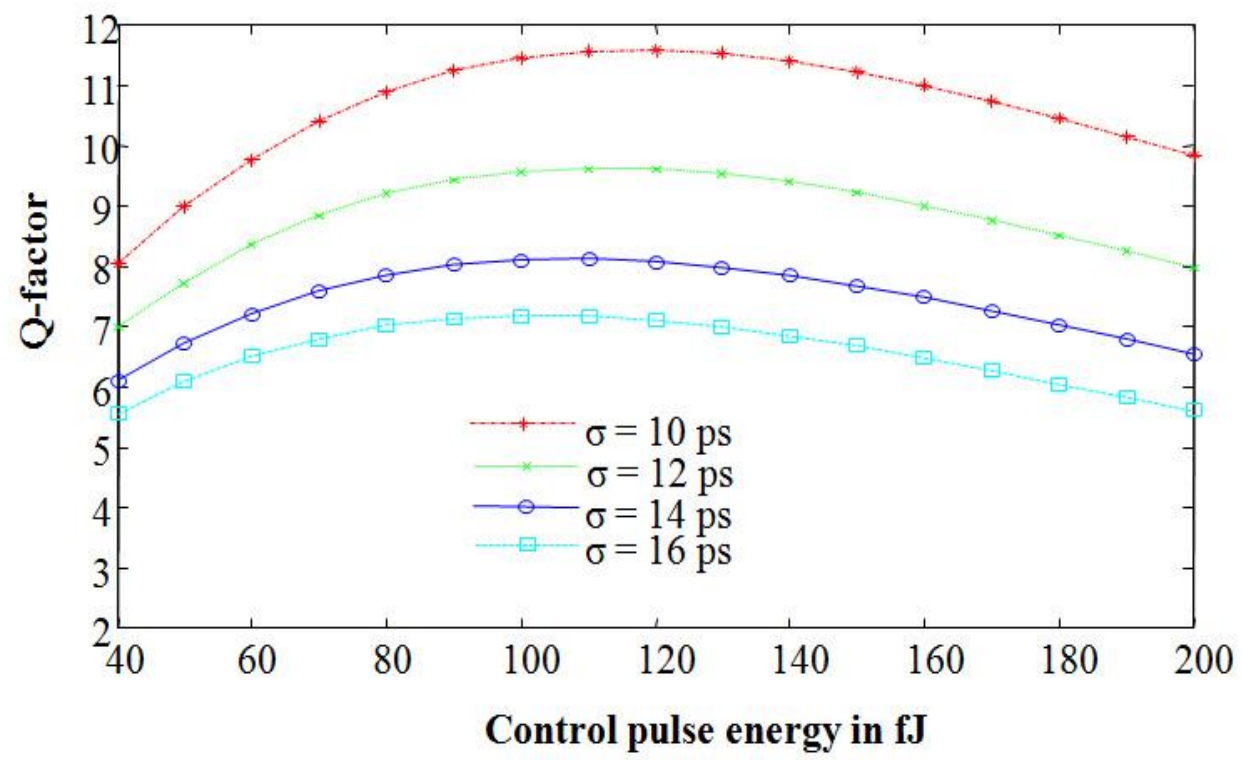

Fig. 9. Variety of Q-factor as a component of energy of $\mathrm{CP}$ with various pulse widths at the various $\mathrm{O} / \mathrm{Ps}$.

From Figs. 5-9 and their clarifications, we select estimations of the different boundaries as $\mathrm{T}_{\mathrm{ex}}=30 \mathrm{ps}, \mathrm{G}_{\mathrm{ss}}=20 \mathrm{~dB}, \alpha=6, \tau_{\mathrm{e}}=100 \mathrm{ps}, \mathrm{E}_{\mathrm{sat}}=1000 \mathrm{fJ}, \mathrm{E}_{\mathrm{cp}}=100 \mathrm{fJ}$ and $\sigma=12 \mathrm{ps}$ individually, which isn't fixed and stays inside the specified limits. At that point utilizing these qualities all through the recreation, we can get $\mathrm{ER}=11.51 \mathrm{~dB}, \mathrm{CR}=15.62 \mathrm{~dB}, \mathrm{AM}=$ $0.05 \mathrm{~dB}$, and $\mathrm{Q}=8.13$ at the $\mathrm{O} / \mathrm{Ps}$. To plan n-bit circuit, we require n-no. of TOADs, $n$ inputs. The circuit has $2 n$-quantities of $\mathrm{O} / \mathrm{Ps}$, among them $n$-output bits addresses 1 's complement results $\left(C_{n}\right.$ to $\left.C_{0}\right)$ and $(n-1)$ bit represents the integer bits $\left(D_{n}\right.$ to $\left.D_{0}\right)$ of the division-by-2 output and 1-bit ( $\left.\mathrm{D}_{-1}\right)$ for fractional bit. 


\section{Conclusion}

We have proposed and mathematically showed a straightforward plan of synchronous alloptical division by 2 and 1s supplement at 20-Gbit/s utilizing TOADs. The circuit is planned hypothetically and verified through mathematical recreations. Here, by leading mathematical recreation, study precise the necessities for the control pulse energy, acquire recuperation time and information pulse width with the goal that the extinction ratio, contrast ratio, amplitude modulation and Q factor is acceptable. In this proposed plot, the extinction ratio is about $11.51 \mathrm{~dB}$, contrast ration is about $15.62 \mathrm{~dB}$, amplitude modulation is about $0.05 \mathrm{~dB}$, and Q-factor is 8.13 at the O/Ps. The benefit of the designed is all-optical in nature and can be stretched out to n-bit without any problem.

\section{References:}

1. C.Ra_aelli, K.Vlachos, N.Andriolli, D.Apostolopoulos, J.Buron, R.V.Caenegem, G.Danilewicz, J.M.Finochietto, J.Garia-Haro, D.Klonidis, M. O'Mahony, G.Maier, A.Pattavina, P.Pavon-Marino, S.Ruepp, M.Savi, M.Sca_ardi, I.Tomkos, A.Tzanakaki, L.Wosinaka, O.Zouraraki and F.Neri, "Photonics in switching: architectures, systems and enabling technologies", Computer Networks, vol.52, pp. 1873-1890, (2008).

2. J. P. Sokoloff, P. R. Prucnal, I. Glesk, and M. Kane, "A terahertz optical asymmetric demultiplexer (TOAD)", IEEE Photonic Technology Letters, vol.5, no.7, pp. 787-789, (1993).

3. K. E. Zoiros, C. S. Koukourlis, and T. Houbavlis, "Analysis and design of ultrahighspeed all-optical semiconductor-optical-ampli_er assisted Sagnac recirculating shift register with an inverter", Optical Engineering, vol.44, no.6, pp.065001-12, (2005).

4. D. K. Gayen, T. Chattopadhyay, M. K. Das, J. N. Roy, and R. K. Pal, "All-optical binary to gray code and gray to binary code conversion scheme with the help of semiconductor optical ampli_er assisted Sagnac switch", IET Circuits, Devices \& Systems, vol.5, no.2, pp.123-131, (2011).

5. S. Sharma and S. Roy, "Design of all-optical parallel multipliers using semiconductor optical amplifier-based Mach-Zehnder interferometers", The Journal of Supercomputing, https://doi.org/10.1007/s11227-020-03543-0 (2021).

6. S K. Maji, K. Mukherjee and A. Raja, "An alternative method for implementation of frequency-encoded logic gates using a terahertz optical asymmetric demultiplexer (TOAD)", Journal of Computational Electronics, 18, 1423-1434 (2019).

7. L.Huo, C.Zhao, H.Wu, and M.Tang, "A Reconfigurable Microwave Photonic Filter Based on Multicore Fibers Incorporating a TOAD Switch", Asia Communications and Photonics Conference/International Conference on Information Photonics and Optical Communications 2020 (ACP/IPOC) OSA Technical Digest (Optical Society of America, 2020), paper M4A.339 $\bullet$ https://doi.org/10.1364/ACPC.2020.M4A.339.

8. K. Maji, K. Mukherjee and M. K. Mandal, "Analysis of All Optical Dual Control Dual SOA TOAD Based 2's Complement Generator," 2020 IEEE VLSI DEVICE CIRCUIT
AND
SYSTEM
(VLSI
DCS),
2020 ,
pp.
119-123, doi:10.1109/VLSIDCS47293.2020.9179909. 
9. K.Maji and K.Mukherjee, "Design and Analysis of X-OR Gate and 4-Bit Binary to 4-Bit Gray and Gray to Binary Code Converter Using Dual Control Dual SOA TOAD (DCDSTOAD)", Micro and Nanosystems, Volume 12, Number 3, 2020, pp. 175-186(12).

10. H. Le Minh, Z.Ghassemlooy and W.P.Ng, "Charecterization and and performance analysis of a TOAD switch employing a dual control pulse scheme in high speed OTDM demultiplexer", IEEE Commun. Letter, vol. 12, no. 4, pp. 316-318, (2008).

11. A.Bhattachryya, D. K. Gayen, and T. Chattopadhyay, "All-optical 4-bit Binary to Binary Coded Decimal Converter with the help of Semiconductor Optical Amplifier assisted Sagnac Switch", Optics Communications, vol.293, pp. 31-42, December (2013).

12. T.Chattopadhyay, "All-optical clocked delay flip-flop using a single terahertz optical asymmetric demultiplexer-based switch: a theoritical study", Applied Optics, vol. 49, no.28, pp. 5226-5235, October (2010).

13. D. K. Gayen, T. Chattopadhyay, A. Bhattacharyya, S. Basak and D. Dey, "All-optical half adder/ subtractor using terahertz optical asymmetric demultiplexer", Applied Optics, vol.53, no.36, pp. 8400-8409, December (2014).

14. M. Eiselt, W. Pieper, and H. G. Weber, SLALOM: Semiconductor laser amplifier in a loop mirror, Journal of lightwave Technology, vol.13, no.10, pp. 2099-2112, (1995).

15. K. E. Zoiros, G. Papadopoulos, T. Houbavlis, and G. T. Kanellos, "Theoretical analysis and performance investigation of ultra-fast all-optical Boolean XOR gate with semiconductor optical amplifier-assisted Sagnac interferometer", Optics Communications, vol.258, no.2, pp. 114-134, (2006).

16. K. E. Zoiros, T. Houbavlis, and M. Kalyvas, "Ultra-high speed all-optical shift registers and their applications in OTDM networks", Optical and Quantum Electronics, vol. 36, no. 11, pp. 1005-1053, (2004).

17. K. E. Zoiros, P. Avramidis, and C. S. Koukourlis, "Performance investigation of semiconductor optical amplifier based ultra-fast nonlinear interferometer in nontrivial switching mode", Optical Engineering, vol. 47, no. 11, pp. 115006-11, (2008) 